\title{
Tecnología
}




\section{El ingeniero industrial y el diseño de máquinas}

Juan Carlos Goñi

Ingeniero mecánico por la Universidad Nacional de Ingeniería. Doctor en ciencias de la ingeniería por la Universidad Federal de Ciencias de Zurich (ETH - Zurich) Suiza. Ingeniero asociado de Alfa-Plus Consultora en Ingeniería.

En todo proceso productivo se necesita seleccionar o diseñar los equipos y máquinas más adecuadas para garantizar los mejores resultados en la empresa. Es por eso que se necesita la participación del ingeniero industrial con el fin de canalizar y dirigir dichos objetivos.

De aquí se desprenden las siguientes interrogantes:

- ¿Qué debe saber un ingeniero industrial sobre diseño de máquinas?

- ¿Cuál es la relación de un ingeniero industrial con el diseño, selección y construcción de un equipo o sistema?

- ¿Qué importancia tiene el mantenimiento de los equipos de una planta y de todas sus instalaciones?

- ¿De qué manera se debe tener actualizada la tecnología de una fábrica, y los procesos productivos de una empresa?

- ¿Qué esperan las empresas de un ingeniero industrial frente al área de diseño de máquinas? 


\section{Presentación}

El concepto general que se tiene de un inventor o de un ingeniero es el de poner en juego su imaginación y el de crear un diseño nuevo. En realidad, aún en el caso de que se cree una máquina antes jamás concebida, se hace uso de ideas ya conocidas desde largo tiempo atrás, y se saca provecho de las experiencias de una o varias industrias. Un diseñador debe saber evaluar mecanismos, conocer la resistencia de materiales y tener los criterios de tolerancia y dimensionamiento que pueden ser aplicados en la creación de máquinas.

Un nuevo modelo de máquina-herramienta es generalmente muy parecido a otro anterior, y un nuevo modelo de automóvil es análogo en muchos aspectos al precedente. Las modificaciones (basadas en la experiencia obtenida con el modelo antiguo) se introducen ya sea con el fin de mejorar la máquina o bien para alcanzar una ventaja económica o mejora técnica.

Un ejemplo interesante de diseño de mecanismos puede representar un extractor de humos de soldadura en el interior de un ambiente cerrado; es decir, una campana extractora con ayuda de aire comprimido, para aprovechar el efecto de succión. Es curioso imaginarse que en nuestro medio no hay la costumbre de soldar bajo techo, y por lo tanto, se cree que hacerlo es una situación absurda y peligrosa, pero no se le da a esta explicación ninguna respuesta técnica, ni de ingeniería, que por lo demás, no existe. En ingeniería todo es posible, sólo hay que crear, inventar y diseñar. Luego se adopta la mejor alternativa, que en este caso es la campana extractora de gases (ver figura). 


\section{Cámara extractora de gas}

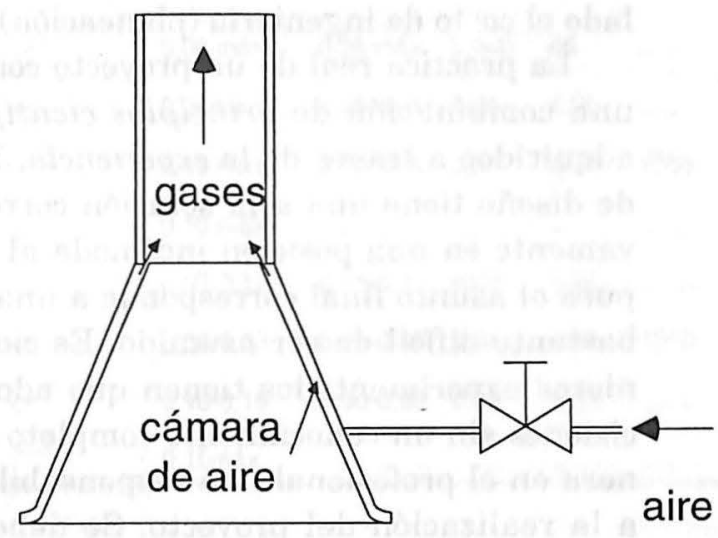

gases de soldadura

Los puntos de vista que tiene un proyectista para la fabricación de un solo producto, por ejemplo lapiceros, son muy diferentes a los que tiene un proyectista para construir automóviles o aviones. Es decir, se necesita consultar a los expertos del área correspondiente, al fabricante del producto en sí y no a conocedores generales. Hay compañías e individuos que pasan su vida entendiendo, desarrollando y fabricando sólo un elemento de máquina. La opinión de estos especialistas es de mucha importancia.

Diseño de máquinas

El motivo principal por el cual se crea una nueva máquina es la existencia de su necesidad presente o previsible. El proceso de creación se inicia con la concepción de un dispositivo, que sirva para una determinada finalidad. A la idea previamente concebida le sigue el estudio de la disposición de las diversas partes y de la posición y longitud de las conexiones, pernos, re- 
sortes, levas y demás componentes de la máquina. Al final se llega a varias soluciones totalmente factibles, y se adopta la más favorable, considerando los criterios económicos (costos), de fabricación, ensamble, operación y mantenimiento, sin dejar de lado el costo de ingeniería (planeación).

La práctica real de un proyecto consiste en la aplicación de una combinación de principios científicos y de conocimientos adquiridos a través de la experiencia. Pocas veces un problema de diseño tiene una sola solución correcta y esto pone definitivamente en una posición incómoda al proyectista principiante, pues el asunto final corresponde a una toma de decisión, punto bastante difícil de ser asumido. Es cierto que incluso los ingenieros experimentados tienen que adoptar frecuentemente decisiones sin un conocimiento completo de la materia, lo que genera en el profesional una responsabilidad anterior y posterior a la realización del proyecto. Se debe tener presente que los trabajos de ingeniería requieren, usualmente, de la adopción de soluciones conciliatorias, de compromiso, pues además del ingeniero intervienen muchas personas más en la toma de decisiones.

En términos generales, un ingeniero de proyectos es quien proyecta -valga la redundancia- un asiento plegable, un bastidor de auto, un modelo de piezas de vajilla de loza o de plata, un lapicero, o un nuevo engranaje, o, incluso, un nuevo sistema de producción.

\section{Construcciones de equipos}

Las decisiones que se tomen para la selección del material de una pieza o parte de un mecanismo se deben basar en el conocimiento de las propiedades mecánicas de los materiales. Las normas técnicas más usadas en la industria para identificar la composición de los materiales son el Sistema SAE (Society of Automotive Engineers) y el Sistema AISI (American Iron and Steel Institute). Los números de especificaciones SAE y AISI son iguales para el acero, excepto en que la AISI utiliza los prefijos B, C, D y E para indicar el método de obtención del acero; (véase tabla adjunta). 


\section{Acero al carbono Bessemer ácido y Siemens básico}

\begin{tabular}{|c|c|c|c|c|c|c|c|c|}
\hline $\begin{array}{l}\text { Número } \\
\text { AISI }\end{array}$ & $\begin{array}{l}\text { Semi- } \\
\text { acabado }\end{array}$ & Barras & $\begin{array}{l}\text { Redondos } \\
\text { para alambre }\end{array}$ & C & $\mathrm{Mn}$ & $\mathrm{Pb}$ & $\mathrm{Sb}$ & $\begin{array}{l}\text { Número } \\
\text { ' } 42 \text { SAE }\end{array}$ \\
\hline C 1005 & & & & 0,06 máx. & 0,35 máx. & 0,040 & 05 & $\ldots .$. \\
\hline C $1006^{*}$ & $Q, R$ & & & 0,08máx. & $0,25-0,40$ & 0,04 & 0,05 & $\ldots$. \\
\hline C $1008^{*}$ & $Q, R$ & & & 0,10 máx. & $0,30-0,50$ & 0,04 & 0,05 & 1008 \\
\hline CB 1008 & & & & 0,10 máx & ………........... & .. & ........ & …......... \\
\hline C 1009 & $Q$ & & & $0,07-0,12$ & $0,25-0,4$ & 0,04 & 0,05 & .......... \\
\hline C $1010^{*}$ & $Q, R$ & & & $0,08-0,13$ & $0,30-0,50$ & 0,04 & 0,05 & 1010 \\
\hline C 1012 & $Q$ & & & $0,10-0,15$ & $0,30-0,50$ & 0,04 & 0,05 & ......... \\
\hline CB 1012 & & & & 0,15máx & .................. & $\ldots \ldots .$. & ......... & ........... \\
\hline
\end{tabular}

* Para material forjado

Un equipo o máquina consta de varias partes mecánicas, como son: un eje o árbol, ya sea para transmitir torque o como soporte, como el caso de los ejes de las ruedas de un vehículo, o ya sea levas para lograr movimiento lineal de vaivén y otros. Es decir, el proceso de manufactura metal-mecánica está fuertemente ligada a la construcción de equipos.

Algunos sistemas que contribuyen a la construcción y ensamble de equipos son:

El torno, para árboles y ejes; el taladro de columna, para hacer agujeros pasantes y ciegos; la fresadora, para canales y engranajes; el cepillo o la limadora, para lograr superficies metálicas planas y en general máquinas-herramientas con arranque de viruta. Otras máquinas pueden ser:

- La troqueladora, para hacer agujeros a planchas metálicas, por acción de impacto y corte.

- La mandrinadora, o también llamada taladradora horizontal.

- La rectificadora, para el ajuste de medida de cilindros y superficies planas.

Como equipos complementarios se tiene: la dobladora de tubos, la cizalladora, la prensa hidráulica, la laminadora; y como sistemas de unión permanente: la soldadura eléctrica, soldadura autógena, MIG, TIG, plasma, arco sumergido y otros. 
Asimismo, se requiere de elementos de medición y comparación, como son los calibradores de medida, micrómetros, verniers, alturímetros, profundímetros, goniómetros, el mármol para comparar superficies, las galgas para controlar luces o huelgo, diámetros de agujeros, medida de roscas arcos, entre otros.

Es imposible fabricar una pieza mecánica con una dimensión exacta, entonces el diseñador debe indicar por lo tanto las cotas máximas y mínimas que pueden ser aceptadas. Para esto, se pueden seguir tanto las normas ASA como las normas ISO. Debemos considerar los siguientes conceptos: medida nominal, tolerancia, desviación, margen, ajuste con juego, ajuste con interferencia y ajuste con apriete.

El dimensionamiento funcional de una pieza es un aspecto de mucha importancia para el éxito de cualquier operación de mecanizado. Así, una pieza en una fresadora debe dimensionarse de tal forma que la mesa se desplace desde una línea de referencia hasta el primer agujero, y desde el primer agujero hasta el segundo, y así sucesivamente. Es decir siempre se debe pensar en el proceso de fabricación de la pieza.

Si la misma pieza se va a perforar en una mandrinadora de precisión, se requiere dimensionar a partir de un vértice, de un punto de referencia o de un agujero. Es decir, en cada uno de los casos considerados las tolerancias difieren, tratando de evitar la acumulación de errores. Nunca se debe dejar al operario el cálculo de alguna dimensión ni la especificación de alguna tolerancia.

Selección de equipos, instrumentos y controles

Cuando se habla de un equipo, por ejemplo una bomba o un motor de combustión interna, existen varias posibilidades de escoger. Se debe hacer un estudio técnico del caso:

- Tomar en cuenta qué producto se va a bombear: agua o aceite, combustible o jugo de frutas, soluciones químicas o sólidos, o para el caso de un motor de combustión interna a cuántas revoluciones por minuto debe girar el eje, el combustible que se va a usar, qué potencia es requerida, si es un motor estacionario o de velocidad variable, y qué niveles de potencia y ruido se esperan y serán admitidos. 
- Se debe considerar en qué zona geográfica se va a montar el equipo: en la selva, en la puna o cerca al mar, con un medio ambiente abrasivo, y si existe en la zona facilidad de servicios de reparación y mantenimiento.

- También se debe observar qué tipo de equipo se requiere, de acuerdo con la carga, sea continua o variable, a la temperatura de trabajo y al número de horas diarias que estará sometida la máquina.

Para la selección de una bomba se debe hacer un estudio de la instalación de bombeo, en la que hay que definir los siguientes parámetros:

- Caudal y altura de bombeo.

- Curva de trabajo del sistema, pérdidas por tuberías y accesorios.

- Naturaleza del fluido a transportar; es decir, viscosidad, densidad, corrosividad, estabilidad química, volatibilidad, cantidad de partículas en suspensión.

- Altura de aspiración y otros.

Para la selección de un motor de combustión interna se deben observar fundamentalmente los factores de aplicación:

- Motor diesel o a gasolina.

- Motor estacionario, para grupos electrógenos, podadoras de césped.

- Motores semiestacionarios, buques, motores fuera de borda.

- Motores de velocidad variable, rápidos y lentos, para la industria automotriz.

- Potencia requerida y otros.

Para la selección de instrumentos de medición y control se requiere:

- Medición de temperatura, rango de trabajo, precisión, lugar de medición.

- Medición de la presión atmosférica, de vacío o manométrica; rango de aplicación, medio que se desea medir.

- Medición del voltaje y la corriente eléctrica, si ésta es alterna o continua, aplicación a motores eléctricos o a sistemas de iluminación.

- Medición y control de la velocidad lineal, rotatoria, o caudal de líquidos o gases, velocímetros, tacómetros, o 
contómetros de agua, surtidores de gasolina, o tanques de estancamiento para medición del flujo de aire.

- Sistemas de control a través de sensores ópticos o sensores de temperatura o presión.

\section{Mantenimiento de las instalaciones y máquinas}

Cuando se toca el tema del mantenimiento se confía muchas veces en la bondad eterna de una máquina. Pero debemos tener en consideración el mantenimiento correctivo, el mantenimiento preventivo, el mantenimiento predictivo y el mantenimiento reactivo. También se debe considerar el mantenimiento productivo total (TPM).

El mantenimiento correctivo solamente solucionará un problema que se debió prever, como es el de rectificar los cilindros de un motor diesel porque se quedó sin aceite; o cambiar los platinos, bujías o filtro de gasolina, porque no se programó el afinamiento de un motor a gasolina.

El mantenimiento preventivo es sumamente importante en una instalación. La falta de orden en una oficina es signo de caos y, por consiguiente, falta de eficiencia en la utilización del tiempo disponible. Por extensión, un archivador es el mantenimiento preventivo para toda la documentación de una entidad $\mathrm{y}$, por lo tanto, la programación del mantenimiento de una instalación es el mantenimiento preventivo del sistema productivo correspondiente.

Una planta de procesamiento de conservas de alimentos, requiere de una pulcritud clínica. Su aspecto es un indicador de la calidad del producto. No se puede permitir trabajar con un concentrado de pasta de tomate y al final del día retirarse sin dejar limpio el sistema. El mantenimiento preventivo también incluye la revisión periódica de las válvulas de carga, descarga, de las bombas de vacío, del motor mezclador, revisar los niveles de aceite en donde corresponde y otros. Esto se puede programar por ejemplo al inicio de cada jornada de trabajo.

En la práctica el mantenimiento condicional es el que se utiliza, pues abarca el mantenimiento preventivo que se programa y ejecuta, y además, cualquier imponderable obligará a un mantenimiento correctivo del sistema. 


\section{Administración y lo gerencia de proyectos}

En el diseño de máquinas el ingeniero industrial juega el papel de administrador o gerente del proyecto. Por proyecto se entiende diseñar y construir un equipo, montar un laboratorio, o una ampliación de planta; o incluso realizar un estudio económico o de mercado de un producto, entre otros.

Las responsabilidades de un ingeniero industrial frente a un proyecto en el área de diseño de máquinas son más fáciles de precisar si se comparan con las responsabilidades que le corresponden a un proyectista de máquinas.

\section{Responsabilidad del proyectista de máquinas}

Un buen proyectista debe poseer muchas aptitudes, como son:

- Conocer bien la teoría de resistencia de materiales. Las diversas partes y piezas de una máquina deben tener la rigidez adecuada, las tolerancias definidas, y los factores de seguridad correctamente establecidos.

- Tener amplio conocimiento de las propiedades de los materiales empleados en las máquinas. Se debe usar acero inoxidable de diferentes calidades para el caso de equipos del área alimentaria, como también hierro de fundición para monoblocks de motores reciprocantes. Se debe hacer una permanente actualización de la información.

- Estar familiarizado con las características técnicas y económicas de los diversos procesos de fabricación. Esto encierra el área de carpintería metálica, que comprende el torneado, fresado, limado, rectificado entre otros. Las piezas que se construyen deben tener precios competitivos que faciliten su puesta en el mercado.

- Conocer los problemas de corrosión a muy bajas temperaturas o también a relativamente elevadas temperaturas. A altas temperaturas se produce la oxidación de algunos metales debido al oxígeno del aire.

- Disponer de un buen sentido de la estética. Existe una regla general en la ingeniería y es que si una pieza mecánica no se encuentra proporcionada, y a simple vista no muestra buena arquitectura, entonces, así los cálcu- 
los de resistencia de materiales indiquen lo contrario, esa pieza no dará buenos resultados. Además, un producto con buen acabado y buena estética facilitará su venta.

- Tener conocimientos de economía y costos comparativos. Todo factor que suponga un aumento de costos debe ser justificado con una mejora del funcionamiento del sistema.

- Poseer una mente intuitiva y creadora. La imaginación y el ingenio juegan un papel importante.

\section{Responsabilidad del administrador o gerente del proyecto}

El proyectista, a este nivel, debe poseer otro tipo de aptitudes, como son:

- Una visión amplia del proyecto. Debe plantear su objetivo inicial e intermedio. Debe tener bien claro cuáles son los objetivos finales que se persiguen. No puede olvidar que la razón de un proyecto se dirige a un consumidor final, que será el cliente, calificador de dicho proyecto.

- Requiere de amplio criterio, poseer cualidades de jefe, quien ordena y toma decisiones sobre puntos que no necesariamente tiene que entender o dominar. Requiere talento en la dirección de personal, y capacidad de trato con todos los niveles sociales.

- Saber medir los avances del proyecto. Un diagrama de barras es fundamental para llevar el control de tiempo.

- Estar capacitado para elaborar un cronograma realista de trabajo. El mayor tiempo empleado en la ejecución de un programa conlleva mayores gastos generales y en muchos casos genera pérdidas por costo de oportunidad o moras, es decir un aumento en los costos indirectos.

- Ubicar a los asesores adecuados en el área de la ingeniería mecánica o el área que corresponda al proyecto. No es necesario ni inteligente tratar de demostrar conocimientos sobre un tema que en realidad uno no conoce. La calidad de un buen ingeniero industrial está en saber elegir el equipo de trabajo que va a llevar adelante su proyecto. 


\section{Bibliografía}

Freirer, John

Maquinado de metales con máquinas herramientas. México: Continental, 1992.

Galarza, Sergio

"Planeamiento estratégico: herramienta para el éxito empresarial". Revista Ingeniería Industrial 17. Lima: Universidad de Lima, Facultad de Ingeniería Industrial, 1996, pp. 23-45.

Liu García, Ángel

"Planta envasadora y distribuidora de gas licuado de petróleo". Revista Ingeniería Industrial 15. Lima: Universidad de Lima, Facultad de Ingeniería Industrial, 1995, pp. 75-91.

Rase/Barrow

Ingeniería de proyecto. México: Continental, 1984.

Reaño Álvarez, José

"Certificación ISO 9000 y sus implicancias". Revista Ingeniería Industrial 15. Lima: Universidad de Lima, Facultad de Ingeniería Industrial, 1995, pp. 92-96.

Slaymaker R.P.

Diseño y análisis de elementos de máquinas. México: Limusa, 1991.

Universidad de Lima

Revista Ingeniería Industrial 15. Lima: Universidad de Lima, Facultad de Ingeniería Industrial, 1995, p. 5. 